\title{
Quantum state transfer in atom-cavity systems with uncolored Cayley interacting networks
}

\author{
R. Sufiani ${ }^{a, b *}$ \\ a Department of Theoretical Physics and Astrophysics, University of Tabriz, Tabriz 51664, Iran. \\ ${ }^{b}$ Institute for Studies in Theoretical Physics and Mathematics, Tehran 19395-1795, Iran.
}

March 27, 2021

*E-mail:sofiani@tabrizu.ac.ir 


\begin{abstract}
Considering the two-photon exchange interaction between $n$ coupled cavities each of them containing a two level atom, the atomic and photonic state transfer is investigated. In fact, $n$ atom-cavity systems are considered to be distributed on the nodes of an uncolored Cayley network and interact with each other via the adjacency matrix of the corresponding network. Then, by employing the photon-excitation conservation and also the algebraic structure of the networks, such as irreducible characters of the groups associated with the networks, some suitable basis for the atom-cavity state space is introduced based on the corresponding generalized Fourier transform, so that the Hamiltonian of the whole system, is block diagonalized with two-dimensional blocks. Then, by solving the corresponding Schrödinger equation exactly, quantum state transfer and also entanglement generation between the atoms or the photons are discussed. For instance, the probability amplitudes associated with the photon transition between the cavities or excitation transition between the atoms are obtained in terms of the irreducibles characters of the corresponding network and the hopping parameter $\xi$ between the cavities.

Keywords: coupled cavities, two-photon exchange, hopping strength, twolevel atoms, generation of entanglement, excitation and photon transfer, uncolored Cayley graphs, Generalized Fourier transform
\end{abstract}

PACs Index: 03.65.Ud 


\section{Introduction}

In the past years, several schemes for quantum information transition and the generation and distribution of entanglement have been designed and implemented in a number of physical systems (see for example [1]-[10]). Atoms and ions are particularly considered as tools for storing quantum information in their internal states. On the other hand, photons represent the best qubit carrier for the fast and reliable communication over long distances $[11,12]$. Recently, using photons in order to achieve efficient quantum information transmission between spatially distant atoms has investigated in several works $[13,14,15,16,17,18]$. The main idea, is to utilize two photon exchange hopping between optical cavities containing the atoms. One of the known models in quantum optics describing the atom-field interaction is the Jaynes-Cummings Hamiltonian [19, 20]. In Refs. [13, 16], entanglement properties of two and three atom-cavities coupled via two-photon exchange interaction, was studied in detail. The motivation of interest to such systems is their ability to be used in quantum switching, quantum communication and computation, and quantum phase transition applications.

In the recent work [18], the approach of Alexanian, et. al has generalized to the case of $n$ atom-cavities - instead of two or three cavities- all of them interacting with each other by hopping strength $\xi$ (in that case the cavities were distributed on a complete network $K_{n}$ at which all of the vertices connected to each other). In this paper, I consider that the atomcavity systems are interacting with each other via the more general connectivity networks (graphs) called uncolored Cayley networks. Due to the fact that these graphs are constructed via finite groups, one can naturally use their algebraic structure in order to state transfer or entanglement generation. In fact, I consider the Cayley graphs associated with abelian groups and employ the group theoretical properties of these networks such as generalized Fourier transform associated with the corresponding groups (the unitary transform which diagonalizes the regular representation of the groups). Then, by using the photon-excitation conservation 
symmetry of the Hamiltonian, some suitable basis for the atom-cavity state space is introduced, so that the Hamiltonian of the whole system, is block diagonalized with two dimensional blocks. Then, by solving the corresponding Schrödinger equation exactly, state transfer between atoms or photons is discussed. For instance, the probability amplitudes associated with the photon transition between the cavities or excitation transition between the atoms are obtained in terms of the irreducibles characters of the groups associated with the corresponding networks, and the hopping parameter $\xi$ between the cavities.

The paper is organized as follows. In section 2, a preliminary is given in order to recall the definition of uncolored Cayley networks. In section 3, the model describing the system of $n$ identical atom-cavities is introduced. The block-diagonalization of the Hamiltonian of the system, solving the corresponding Shrödinger equation for time dependent probability amplitudes of the state of the system, and entanglement generation between atoms or photons are discussed in this section. Section 4 is devoted to discussions about state transfer (excitation or photon transfer between the atoms or the cavities). In sections 5, two examples of Cayley networks (Cycle network $C_{n}$ and the hypercube network $Q_{d}$ ) are discussed in detail, in order to clarify the results of the paper. The paper is ended with a brief conclusion.

\section{Preliminary: Uncolored Cayley networks}

For a given finite group $G$, we have always a set of group elements $S$ so that all of the group elements can be generated via the possible multiplications of the elements of $S$ and their powers. The set $S$ is called the generating set of $G$. The Cayley graph $\Gamma(G, S)$ is a colored directed graph constructed as follows: Each element $g$ of $G$ is assigned a vertex: the vertex set $V(\Gamma)$ of $\Gamma$ is then identified with $G$. Each generator $s$ of $S$ is assigned a color $c_{s}$. For any $g \in G$ and $s \in S$, the vertices corresponding to the elements $g$ and $g s$ are joined by a directed edge of color $c_{s}$. Thus the edge set $E(\Gamma)$ consists of pairs of the form $(g, g s)$ with $s \in S$ providing 
the color.

The set $S$ is usually assumed to be finite, symmetric (i.e. $S=S^{-1}$ ) and not containing the identity element of the group. In this case, the uncolored Cayley graph is an ordinary graph: its edges are not oriented and it does not contain loops. Then, the adjacency matrix $A$ corresponding to the Cayley graph $\Gamma(G, S)$ is defined as:

$$
A=\sum_{s \in S} R(s)
$$

where $R(s)$ is the regular representation of the group element $s \in S$.

We will assume the Cayley graphs corresponding to the Abelian group, so that the adjacency matrix $A$ can be diagonalized via generalized Fourier transform $P$ defined by $P_{i j}=$ $\frac{1}{\sqrt{n}} \chi_{i}(j)$ where, $\chi_{i}(j)$, for $i, j=1,2, \ldots, n(=|G|)$, is the $i$-th irreducible character of the $j$-th group element, and $|G|$ is the cardinality of the group. Then, the eigenvalues of the adjacency matrices $A_{k}$ are given by

$$
x_{i}=\sum_{s \in S} \chi_{i}(s)
$$

It could be noticed that, from the orthogonality relation for the irreducible characters [21],

$$
\frac{1}{n} \sum_{k} \chi_{i}(k) \chi_{j}^{*}(k)=\delta_{i j}
$$

one can see that $\left(P^{-1}\right)_{i j}=\frac{1}{\sqrt{n}} \chi_{j}^{*}(i)$ and so we have $P^{-1}=P^{\dagger}$.

\section{The Model: $n$ coupled cavities via two-photon ex- change interaction}

We will consider $n$ identical cavities each containing a two-level atom, where the cavities are coupled via two photon hopping between them. In fact, we consider that the cavities are located at the nodes of an uncolored Cayley network with $n$ nodes and each cavity interacts with all of the adjacent cavities, via two-photon exchange. 
Let us first introduce the Hamiltonian of one individual atom-cavity as a two-photon JCM $[22]$ (assume $\hbar=1)$ :

$$
H^{(i)}=\omega_{a} s_{z}^{(i)}+\omega_{c} a_{i}^{\dagger} a_{i}+\lambda\left(\sigma_{e g}^{(i)} a_{i}^{2}+\sigma_{g e}^{(i)} a_{i}^{\dagger 2}\right),
$$

where, the operators $a_{i}$ and $a_{i}^{\dagger}$ are photonic annihilation and creation operators of the $i$-th cavity, $\sigma_{a b}^{(i)}=|a\rangle^{(i)(i)}\langle b|$, for $i=1,2, \ldots, n$ denote the atomic transition operators for the $i$ th cavity referring to either the ground (g) or excited (e) state; $\omega_{a}$ is the energy separation between two levels $|g\rangle$ and $|e\rangle, s_{z}^{(i)}=\frac{1}{2} \sigma_{z}^{(i)}=\frac{1}{2}\left(|e\rangle^{(i)(i)}\langle e|-| g\rangle^{(i)(i)}\langle g|\right)$, and $\omega_{c}$ is the resonance frequency of the cavity.

In the Hamiltonian (3-4), the excitation-number operator

$$
\hat{N}^{(i)}=a_{i}^{\dagger} a_{i}+s_{z}^{(i)}
$$

is a constant of motion for the $i$-th atom-cavity subsystem, i.e, we have $\left[H^{(i)}, \hat{N}^{(i)}\right]=0$ for each $i=1,2, \ldots, n$. Now, the Hamiltonian for the $n$ cavities is given by:

$$
H=\sum_{i=1}^{n} H^{(i)}+\xi \sum_{i, j=1 ; i \sim j}^{n}\left(a_{i}^{2 \dagger} a_{j}^{2}+a_{j}^{2 \dagger} a_{i}^{2}\right),
$$

where, $i \sim j$ means that the cavities $i$ and $j$ are adjacent on the network. The last term in the Hamiltonian (3-5) is the two-photon exchange interaction between the cavities, characterized by the hopping rate $\xi$.

The operator $\hat{N}=\sum_{i=1}^{n} \hat{N}^{(i)}$ commutes with the Hamiltonian (3-5) and so we can reduce the Hamiltonian to the subspace spanned with the eigenstates of $\hat{N}$ and consider the time evolution of the states in this subspace. For a given eigenspace of $\hat{N}$ with eigenvalue $N$, the maximum possible number of photons in a cavity is $N$ when the corresponding atom is in the ground state, which occurs when there are no photons present in the other cavities and the atoms are also in the ground state. Then, the total number of photons in the system will be $N$. The constant number of total photons determines the subspace or the manifold in which the states evolve in time (the initial state of the system determines the constant number $N$ ). For 
simplicity, we will consider the manifold with $N=\frac{2-n}{2}$. In this case, each single atom-cavity system can take one of the three possible states $|g, 0\rangle,|g, 2\rangle$ or $|e, 0\rangle$, and so, the total possible states that the system of $n$-cavities can take, are $3^{n}$ states. Due to the consistency of total $N=\frac{2-n}{2}$, the only possible states which we can have, are $2 n$ states instead of $3^{n}$ ones. In fact, these $2 n$ states are eigenstates of $\hat{N}$ with eigenvalue 2 , and the $3^{n}$-dimensional Hamiltonian $H$ is reduced to $2 n$-dimensional one in the bases which span the eigenspace of $\hat{N}$ with the corresponding eigenvalue 2. The bases states that span this subspace or manifold, are given by:

$$
\begin{aligned}
& \left|c_{i}\right\rangle=|g, 0\rangle \ldots|g, 0\rangle \underbrace{|g, 2\rangle}_{i-t h}|g, 0\rangle \ldots|g, 0\rangle, \\
& \left|a_{i}\right\rangle=|g, 0\rangle \ldots|g, 0\rangle \underbrace{|e, 0\rangle}_{i-t h}|g, 0\rangle \ldots|g, 0\rangle,
\end{aligned}
$$

for $i=0,1, \ldots, n-1$. Indeed, these bases span the eigenspace of $\hat{N}$ with eigenvalue 2 , i.e., we have $\hat{N}\left(\alpha\left|c_{i}\right\rangle+\beta\left|a_{i}\right\rangle\right)=\frac{2-n}{2}\left(\alpha\left|c_{i}\right\rangle+\beta\left|a_{i}\right\rangle\right)$. Therefore, the general time dependent state of the $n$-cavity system is given by

$$
|\psi(t)\rangle=\sum_{i=0}^{n-1}\left(C_{i}(t)\left|c_{i}\right\rangle+A_{i}(t)\left|a_{i}\right\rangle\right)
$$

Then, one can easily show that

$$
\begin{gathered}
\sum_{i=1}^{n} H^{(i)}\left|a_{k}\right\rangle=\frac{2-n}{2} \omega_{a}\left|a_{k}\right\rangle+\sqrt{2} \lambda\left|c_{k}\right\rangle, \\
\sum_{i=1}^{n} H^{(i)}\left|c_{k}\right\rangle=\frac{4 \omega_{c}-n \omega_{a}}{2}\left|c_{k}\right\rangle+\sqrt{2} \lambda\left|a_{k}\right\rangle, \\
H_{\text {int. }}\left|c_{k}\right\rangle \equiv \xi \sum_{i, j=1 ; i \sim j}^{n}\left(a_{i}^{2 \dagger} a_{j}^{2}+a_{j}^{2 \dagger} a_{i}^{2}\right)\left|c_{k}\right\rangle=2 \xi \sum_{i \sim k}\left|c_{i}\right\rangle, \quad H_{\text {int. }}\left|a_{k}\right\rangle=0 ; \quad k=0,1, \ldots, n-1 .
\end{gathered}
$$

Now, by considering the order of bases as $\left|c_{0}\right\rangle,\left|a_{0}\right\rangle, \ldots,\left|c_{n-1}\right\rangle,\left|a_{n-1}\right\rangle$, and defining $\Delta=\omega_{a}-2 \omega_{c}$, the Hamiltonian $H$ takes the following direct product form

$$
H=I_{n} \otimes\left(\begin{array}{cc}
\omega+\Delta / 2 & \sqrt{2} \lambda \\
\sqrt{2} \lambda & \omega-\Delta / 2
\end{array}\right)+2 \xi A \otimes\left(\begin{array}{cc}
1 & 0 \\
0 & 0
\end{array}\right) \text {, }
$$


where, $\omega:=\frac{1}{2}\left[(2-n) \omega_{a}-\Delta\right]$ and $A$ is the adjacency matrix of the corresponding network containing the atom-cavities on its nodes. We will assume that the cavities are interacting with each others via an uncolored Cayley network as connectivity network, so that $A \equiv A_{1}$ is given by (2-1). That is, the photons only hope between the cavities which are adjacent in the network.

Now, due to the fact that the adjacency matrix $A$ is diagonalized by the generalized Fourier transform $P_{k l}:=\frac{1}{\sqrt{n}} \chi_{k}\left(\alpha_{l}\right)$, one can naturally consider the new transformed bases $\left\{\left|c_{j}\right\rangle^{\prime},\left|a_{j}\right\rangle^{\prime}\right\}_{j=0}^{n-1}$ as:

$$
\begin{aligned}
\left|c_{j}\right\rangle^{\prime} & :=\frac{1}{\sqrt{n}} \sum_{i=0}^{n-1} \chi_{j}\left(\alpha_{i}\right)\left|c_{i}\right\rangle, \\
\left|a_{j}\right\rangle^{\prime} & :=\frac{1}{\sqrt{n}} \sum_{i=0}^{n-1} \chi_{j}\left(\alpha_{i}\right)\left|a_{i}\right\rangle .
\end{aligned}
$$

Then, by considering the ordering $\left\{\left|c_{0}\right\rangle^{\prime},\left|a_{0}\right\rangle^{\prime} ; \ldots ;\left|c_{n-1}\right\rangle^{\prime},\left|a_{n-1}\right\rangle^{\prime}\right\}$, the Hamiltonian (3-7) takes the following block diagonalized form:

$$
H=I_{n} \otimes\left(\begin{array}{cc}
\omega+\Delta / 2 & \sqrt{2} \lambda \\
\sqrt{2} \lambda & \omega-\Delta / 2
\end{array}\right)+2 \xi \operatorname{diag}\left(x_{0}, x_{1}, \ldots, x_{n-1}\right) \otimes\left(\begin{array}{cc}
1 & 0 \\
0 & 0
\end{array}\right),
$$

where, $\operatorname{diag}\left(x_{0}, x_{1}, \ldots, x_{n-1}\right)$ is the $n \times n$ diagonal matrix with diagonal entries as $x_{i}=\chi_{i}\left(\alpha_{1}\right)$ with $i=0,1, \ldots, n-1$. Therefore, the $i$-th block of the Hamiltonian is given by

$$
H^{(i)}=\left(\begin{array}{cc}
\omega+\Delta / 2+2 \xi x_{i} & \sqrt{2} \lambda \\
\sqrt{2} \lambda & \omega-\Delta / 2
\end{array}\right) .
$$

with eigenvalues $E_{ \pm}^{(i)}=\left(\omega+\xi x_{i}\right) \pm \sqrt{2 \lambda^{2}+\left(\frac{\Delta}{2}+\xi x_{i}\right)^{2}}$. Now, by using the Schrödinger equation of motion $i \hbar \frac{\partial}{\partial t}|\psi\rangle=H|\psi\rangle$, the equations of motion are given by:

$$
\begin{aligned}
i \dot{C}_{i}^{\prime} & =\left[\omega+\Delta / 2+2 \xi x_{i}\right] C_{i}^{\prime}+\sqrt{2} \lambda A_{i}^{\prime} ; \\
i \dot{A}_{i}^{\prime} & =\sqrt{2} \lambda C_{i}^{\prime}+(\omega-\Delta / 2) A_{i}^{\prime},
\end{aligned}
$$

for $i=0,1, \ldots, n-1$. Solving the corresponding differential equations, one can obtain

$$
C_{i}^{\prime}(t)=\frac{e^{-i\left(\omega+\xi x_{i}\right) t}}{\sqrt{2 \lambda^{2}+\left(\frac{\Delta}{2}+\xi x_{i}\right)^{2}}}\left\{\left[\sqrt{2 \lambda^{2}+\left(\frac{\Delta}{2}+\xi x_{i}\right)^{2}} \cos t \sqrt{2 \lambda^{2}+\left(\frac{\Delta}{2}+\xi x_{i}\right)^{2}}-\right.\right.
$$


coupled cavities

$$
\begin{gathered}
\left.\left.i\left(\frac{\Delta}{2}+\xi x_{i}\right) \sin t \sqrt{2 \lambda^{2}+\left(\frac{\Delta}{2}+\xi x_{i}\right)^{2}}\right] C_{i}^{\prime}(0)-i \sqrt{2} \lambda \sin t \sqrt{2 \lambda^{2}+\left(\frac{\Delta}{2}+\xi x_{i}\right)^{2}} A_{i}^{\prime}(0)\right\} \\
A_{i}^{\prime}(t)=\frac{e^{-i\left(\omega+\xi x_{i}\right) t}}{\sqrt{2 \lambda^{2}+\left(\frac{\Delta}{2}+\xi x_{i}\right)^{2}}}\left\{\left[\sqrt{2 \lambda^{2}+\left(\frac{\Delta}{2}+\xi x_{i}\right)^{2}} \cos t \sqrt{2 \lambda^{2}+\left(\frac{\Delta}{2}+\xi x_{i}\right)^{2}}+\right.\right. \\
\left.\left.i\left(\frac{\Delta}{2}+\xi x_{i}\right) \sin t \sqrt{2 \lambda^{2}+\left(\frac{\Delta}{2}+\xi x_{i}\right)^{2}}\right] A_{i}^{\prime}(0)-i \sqrt{2} \lambda \sin t \sqrt{2 \lambda^{2}+\left(\frac{\Delta}{2}+\xi x_{i}\right)^{2}} C_{i}^{\prime}(0)\right\}
\end{gathered}
$$

In the resonance case $\Delta=0$, by considering $\lambda=\frac{1}{\sqrt{2}}$, the coefficients (3-12) read as

$$
\begin{aligned}
& C_{i}^{\prime}(t)=\frac{e^{-i\left(\omega+\xi x_{i}\right) t}}{\sqrt{1+\left(\xi x_{i}\right)^{2}}}\left\{\left[\sqrt{1+\left(\xi x_{i}\right)^{2}} \cos t \sqrt{1+\left(\xi x_{i}\right)^{2}}-i \xi x_{i} \sin t \sqrt{1+\left(\xi x_{i}\right)^{2}}\right] C_{i}^{\prime}(0)-i \sin t \sqrt{1+\left(\xi x_{i}\right)^{2}} A_{i}^{\prime}(0)\right\} \\
& A_{i}^{\prime}(t)=\frac{e^{-i\left(\omega+\xi x_{i}\right) t}}{\sqrt{1+\left(\xi x_{i}\right)^{2}}}\left\{\left[\sqrt{1+\left(\xi x_{i}\right)^{2}} \cos t \sqrt{1+\left(\xi x_{i}\right)^{2}}+i \xi x_{i} \sin t \sqrt{1+\left(\xi x_{i}\right)^{2}}\right] A_{i}^{\prime}(0)-i \sin t \sqrt{1+\left(\xi x_{i}\right)^{2}} C_{i}^{\prime}(0)\right\} .
\end{aligned}
$$

By using (3-8), one can obtain the time dependence of the coefficients $C_{i}(t)$ and $A_{i}(t)$ of the state of the system in (3-6) via the inverse $P$ transform as,

$$
\begin{aligned}
& C_{i}(t)=\frac{1}{\sqrt{n}} \sum_{j=0}^{n-1} \chi_{j}^{*}\left(\alpha_{i}\right) C_{j}^{\prime}(t), \\
& A_{i}(t)=\frac{1}{\sqrt{n}} \sum_{j=0}^{n-1} \chi_{j}^{*}\left(\alpha_{i}\right) A_{j}^{\prime}(t),
\end{aligned}
$$

where, $*$ denotes the complex conjugate. It should be pointed out that, for the probabilities associated with the state of the system as a superposition of atomic states $\left|a_{i}\right\rangle$, and that of photonic states $\left|c_{i}\right\rangle$ (denoted by $P_{a}(t)$ and $P_{c}(t)$, respectively), the equation (3-14) gives

$$
\begin{aligned}
P_{a} & =\sum_{i=0}^{n-1}\left|A_{i}(t)\right|^{2}=\frac{1}{n} \sum_{j, k} \underbrace{\sum_{i} \chi_{j}^{*}\left(\alpha_{i}\right) \chi_{k}\left(\alpha_{i}\right)}_{n \delta_{j k}} A_{j}^{\prime}(t) A_{k}^{\prime}(t)=\sum_{j=0}^{n-1}\left|A_{j}^{\prime}(t)\right|^{2} \\
P_{c} & =\sum_{i=0}^{n-1}\left|C_{i}(t)\right|^{2}=\frac{1}{n} \sum_{j, k} \underbrace{\sum_{i} \chi_{j}^{*}\left(\alpha_{i}\right) \chi_{k}\left(\alpha_{i}\right)}_{n \delta_{j k}} C_{j}^{\prime}(t) C_{k}^{\prime}(t)=\sum_{j=0}^{n-1}\left|C_{j}^{\prime}(t)\right|^{2}
\end{aligned}
$$

For instance, considering the initial state

$$
|\psi(0)\rangle=\frac{1}{\sqrt{n}}(|g, 2\rangle|g, 0\rangle \ldots|g, 0\rangle+|g, 0\rangle|g, 2\rangle|g, 0\rangle \ldots|g, 0\rangle+\ldots+|g, 0\rangle \ldots|g, 0\rangle|g, 2\rangle)=
$$




$$
|g g \ldots g\rangle \otimes \frac{1}{\sqrt{n}}(|200 \ldots 0\rangle+|020 \ldots 0\rangle+\ldots+|0 \ldots 002\rangle)
$$

in which the atoms are initially unentangled, whereas the photons are in a multipartite entangled $W$-state. Then, the initial conditions are given by $A_{j}(0)=0$ and $C_{j}(0)=\frac{1}{\sqrt{n}}$ for all $j=0,1, \ldots, n-1$, and we obtain via equation $(3-8), A_{i}^{\prime}(0)=0$, and $C_{i}^{\prime}(0)=\frac{1}{n} \sum_{j} \chi_{i}\left(\alpha_{j}\right)$ for all i. Then, with the aid of Eq. (3-12), we obtain

$$
\begin{gathered}
P_{a}(t)=\sum_{i=0}^{n-1}\left|A_{i}^{\prime}(t)\right|^{2}=\sum_{i} \sum_{j, k} \frac{2 \lambda^{2} \sin ^{2} t \sqrt{2 \lambda^{2}+\left(\frac{\Delta}{2}+\xi x_{i}\right)^{2}}}{n^{2}\left[2 \lambda^{2}+\left(\frac{\Delta}{2}+\xi x_{i}\right)^{2}\right]} \chi_{i}^{*}\left(\alpha_{j}\right) \chi_{i}\left(\alpha_{k}\right), \\
P_{c}(t)=1-P_{a}(t) .
\end{gathered}
$$

The above result indicates that, in the limit of large $\xi \rightarrow \infty$, we have $P_{c}(t) \simeq 1$ for every time $t$, i.e., for large enough $\xi$, all of the atoms will be at their ground state $|g\rangle$ at every time $t$.

In particular, at the resonance case $\Delta=0$, we obtain

$$
P_{a}(t)=\sum_{i} \sum_{j, k} \frac{2 \lambda^{2} \sin ^{2} t \sqrt{2 \lambda^{2}+\xi^{2} x_{i}^{2}}}{n^{2}\left[2 \lambda^{2}+\xi^{2} x_{i}^{2}\right]} \chi_{i}^{*}\left(\alpha_{j}\right) \chi_{i}\left(\alpha_{k}\right),
$$

where by taking $\lambda=\frac{1}{\sqrt{2}}$, we have

$$
P_{a}(t)=\sum_{i} \sum_{j, k} \frac{\sin ^{2} t \sqrt{1+\xi^{2} x_{i}^{2}}}{n^{2}\left[1+\xi^{2} x_{i}^{2}\right]} \chi_{i}^{*}\left(\alpha_{j}\right) \chi_{i}\left(\alpha_{k}\right) .
$$

From the above result it can be seen that for small hopping strength $\xi \ll$, we have

$$
P_{a}(t)=\frac{\sin ^{2} t}{n^{2}} \sum_{j, k} \underbrace{\sum_{i} \chi_{i}^{*}\left(\alpha_{j}\right) \chi_{i}\left(\alpha_{k}\right)}_{n \delta_{j k}}=\sin ^{2} t,
$$

which indicates that for times $T=\frac{(2 k+1) \pi}{2}$, we have $P_{a}(t)=1$, whereas for times $T=l \pi$, we have $P_{c}(t)=1$, for any $k, l \in Z$. In other words, for small hopping $\xi$, the initial state $(3-15)$ evolves as

$$
\left|\psi\left(\frac{\pi}{2}\right)\right\rangle=\left(\frac{-i}{\sqrt{n}} \sum_{i=1}^{n}|g \ldots g \underbrace{e}_{i} g \ldots g\rangle\right) \otimes|00 \ldots 0\rangle
$$

after time $T=\frac{\pi}{2}$ for which we have $A_{i}\left(\frac{\pi}{2}\right)=\frac{-i}{\sqrt{n}}$ for all $i$, and so the initially unentangled atoms become entangled and the initial photonic $W$-state oscillates between atomic and photonic $W$-states periodically, with time period $\frac{\pi}{2}$. In order to evaluate the amount of quantum 
entanglement between two atoms labeled by $l$ and $m$, one can calculate the state of the corresponding two atoms as

$$
\begin{gathered}
\rho_{a t}^{l m}\left(\frac{\pi}{2}\right)=\operatorname{Tr}_{p h ; k \neq l, m}\left(\left|\psi\left(\frac{\pi}{2}\right)\right\rangle\left\langle\psi\left(\frac{\pi}{2}\right)\right|\right)=\left(1-\frac{2}{n}\right)|g g\rangle\langle g g|+\frac{1}{n}(|e g\rangle\langle e g|+ \\
|g e\rangle\langle g e|+| e g\rangle\langle g e|+| g e\rangle\langle e g|),
\end{gathered}
$$

where the partial trace is taken over photonic states and also over the atomic states corresponding to the atoms other than the atoms $l$ and $m$. Now, the Peres-Horodecki criteria $[23,24]$ known also as positive partial transpose (PPT) criteria can be checked. The partial transpose of $\rho_{a t}^{l m}\left(\frac{\pi}{2}\right)$ with respect to the first subsystem is given by

$$
\left[\rho_{a t}^{l m}\left(\frac{\pi}{2}\right)\right]^{T_{1}}=\frac{1}{n}\left(\begin{array}{cccc}
n-2 & 0 & 0 & 1 \\
0 & 1 & 0 & 0 \\
0 & 0 & 1 & 0 \\
1 & 0 & 0 & 0
\end{array}\right)
$$

so that the corresponding eigenvalues $\lambda_{i}$ are given by $\frac{1}{n}$ (with double degeneracy) and $\lambda_{ \pm}=$ $\frac{1}{2 n}\left(n-2 \pm \sqrt{(n-2)^{2}+4}\right)$, and the negativity is then $N\left(\rho_{a t}^{l m}\left(\frac{\pi}{2}\right)\right)=\sum_{i}\left|\lambda_{i}\right|-1=-2 \lambda_{-}=$ $\frac{1}{n}\left[\sqrt{(n-2)^{2}+4}-(n-2)\right]$. Then, for the case of two cavities, $n=2$, we have $N\left(\rho_{a t}\left(\frac{\pi}{2}\right)\right)=1$ and the initially unentangled atoms, become maximally entangled at times $T=\frac{(2 k+1) \pi}{2}, k \in Z$.

\section{Photon and excitation transfer between the atom- cavity systems}

\subsection{Photon transition}

Hereafter, we consider the resonance case $\Delta=0$ and take $\lambda=\frac{1}{\sqrt{2}}$. In order to investigate photon transition between the cavities, we consider the initial state $|\psi(0)\rangle=|g, 2\rangle|g, 0\rangle \ldots|g, 0\rangle$, with initial conditions $A_{j}(0)=0, C_{0}(0)=1$ and $C_{l}(0)=0$ for all $j=0,1, \ldots, n-1$ and 
$l=1, \ldots, n-1$. Then, one can obtain via equation $(3-8), A_{j}^{\prime}(0)=0$, and $C_{j}^{\prime}(0)=\frac{1}{\sqrt{n}} \chi_{j}\left(\alpha_{0}\right)=$ $\frac{d_{j}}{\sqrt{n}}=\frac{1}{\sqrt{n}}$ for every $j$. Now, with the aid of Eqs. (3-13) and (3-14), we obtain

$$
\begin{aligned}
& C_{i}(t)=\frac{1}{n} \sum_{j} \frac{e^{-i\left(\omega+\xi x_{j}\right) t}}{\sqrt{\left[1+\left(\xi x_{j}\right)^{2}\right]}}\left[\sqrt{1+\left(\xi x_{j}\right)^{2}} \cos t \sqrt{1+\left(\xi x_{j}\right)^{2}}-i \xi x_{j} \sin t \sqrt{1+\left(\xi x_{j}\right)^{2}}\right] \chi_{j}^{*}\left(\alpha_{i}\right), \\
& A_{i}(t)=\frac{-i}{n} \sum_{j} \frac{e^{-i\left(\omega+\xi x_{j}\right) t}}{\sqrt{\left[1+\left(\xi x_{j}\right)^{2}\right]}} \sin t \sqrt{1+\left(\xi x_{j}\right)^{2}} \chi_{j}^{*}\left(\alpha_{i}\right) .
\end{aligned}
$$

\section{A. Small hopping $\xi \ll$}

The above result indicates that for small hopping $\xi \ll$, we have

$$
\begin{gathered}
C_{i}(t) \simeq \frac{e^{-i \omega t} \cos t}{n} \sum_{j} \chi_{j}^{*}\left(\alpha_{i}\right), \\
A_{i}(t) \simeq \frac{-i e^{-i \omega t} \sin t}{n} \sum_{j} \chi_{j}^{*}\left(\alpha_{i}\right) .
\end{gathered}
$$

It would be noticed that from the orthogonality relation $\sum_{i} \chi_{j}^{*}\left(\alpha_{i}\right) \chi_{k}\left(\alpha_{i}\right)=n \delta_{j, k}$, we have clearly

$$
\sum_{i}\left(\left|C_{i}(t)\right|^{2}+\left|A_{i}(t)\right|^{2}\right)=\frac{1}{n^{2}} \sum_{j, k} \sum_{i} \chi_{j}^{*}\left(\alpha_{i}\right) \chi_{k}\left(\alpha_{i}\right)=1 .
$$

The result (5-21) indicates that, at times $T=k \pi$, with $k \in Z$, we have $A_{i}(T)=0$ and the probability of transfer of two photons from the first cavity to the $i$-th cavity is given by

$$
P_{i}(T)=\left|C_{i}(T)\right|^{2}=\frac{1}{n^{2}} \sum_{j, k} \chi_{j}^{*}\left(\alpha_{i}\right) \chi_{k}\left(\alpha_{i}\right) .
$$

\section{B. Large hopping $\xi \gg$}

For large hopping strength $\xi \rightarrow \infty$, the result (4-17) leads to

$$
\begin{aligned}
& C_{i}(t) \simeq \frac{e^{-i \omega t}}{n}\left\{\cos t \sum_{j ; x_{j}=0} \chi_{j}^{*}\left(\alpha_{i}\right)+\sum_{j ; x_{j} \neq 0} e^{-2 i \xi t x_{j}} \chi_{j}^{*}\left(\alpha_{i}\right)\right\}, \\
& A_{i}(t) \simeq \frac{-i e^{-i \omega t}}{n}\left\{\sin t \sum_{j ; x_{j}=0} \chi_{j}^{*}\left(\alpha_{i}\right)+\sum_{j ; x_{j} \neq 0} \frac{e^{-i \xi t x_{j}} \sin \left(\xi t x_{j}\right)}{\xi x_{j}} \chi_{j}^{*}\left(\alpha_{i}\right)\right\} \simeq \frac{-i e^{-i \omega t}}{n} \sin t \sum_{j ; x_{j}=0} \chi_{j}^{*}\left(\alpha_{i}\right) .
\end{aligned}
$$

Again, at times $T=k \pi$, with $k \in Z$, we have $A_{i}(T) \simeq 0$ and the initially unentangled atoms remain unentangled. 


\subsection{Excitation transition}

The excitation transfer between the atoms, can be achieved by preparing the system initially at the state $|\psi(0)\rangle=|e, 0\rangle|g, 0\rangle \ldots|g, 0\rangle$, with initial conditions $C_{j}(0)=0, A_{0}(0)=1$ and $A_{l}(0)=0$ for all $j=0,1, \ldots, n-1$ and $l=1, \ldots, n-1$. Then, one can obtain via equation

$(3-8), C_{j}^{\prime}(0)=0$, and $A_{j}^{\prime}(0)=\frac{1}{\sqrt{n}}$ for every $j$. Again, with the aid of Eqs. (3-13) and (3-14), one can obtain

$$
\begin{aligned}
C_{i}(t) & =\frac{-i}{n} \sum_{j} \frac{e^{-i\left(\omega+\xi x_{j}\right) t}}{\sqrt{\left[1+\left(\xi x_{j}\right)^{2}\right]}} \sin t \sqrt{1+\left(\xi x_{j}\right)^{2}} \chi_{j}^{*}\left(\alpha_{i}\right), \\
A_{i}(t) & =\frac{1}{n} \sum_{j} \frac{e^{-i\left(\omega+\xi x_{j}\right) t}}{\sqrt{\left[1+\left(\xi x_{j}\right)^{2}\right]}}\left[\sqrt{1+\left(\xi x_{j}\right)^{2}} \cos t \sqrt{1+\left(\xi x_{j}\right)^{2}}+i \xi x_{j} \sin t \sqrt{1+\left(\xi x_{j}\right)^{2}}\right] \chi_{j}^{*}\left(\alpha_{i}\right) .
\end{aligned}
$$

Similar to the photon transition (as argued in the previous subsection), one can discuss excitation transfer for small and large hopping limits, where it is seen that at the limiting cases $\xi \ll$ and $\xi \gg$, we have $C_{i}(T) \simeq 0$ for times $T=l \pi$, with $l \in Z$; The probability of transfer of atomic excitation from the first atom to the $i$-th atom, i.e. $P_{i}(T)=\left|A_{i}(T)\right|^{2}$, can be evaluated similarly.

\section{$5 \quad$ Examples}

In this section we give two examples of uncolored Cayley networks for which the photonic and atomic state transfer between atom-cavities is investigated in details.

\section{The Cycle network $C_{n}$}

If $G=Z_{n}$ is the finite cyclic group of order $\mathrm{n}$ and the set $S$ consists of two elements, the standard generator of $G$ and its inverse, i.e., $a, a^{-1}$ with $a^{n}=1$, then the Cayley graph is the cycle $C_{n}$. In this case the irreducible characters of the group are given by $\chi_{k}\left(a^{l}\right)=\omega^{k l}$, for $k, l=0,1, \ldots, n-1$, where $\omega:=e^{\frac{2 \pi i}{n}}$ is the $n$-th root of identity.

The adjacency matrix of the network is $A=S+S^{-1}$, where $S$ is the shift matrix of order 
$n$ so that we have $S^{n}=I$. Then, the corresponding eigenvalues of $A$ are given by $x_{l}=$ $\chi_{l}(a)+\chi_{l}\left(a^{-1}\right)=\omega^{l}+\omega^{-l}=2 \cos \left(\frac{2 \pi l}{n}\right), l=0,1, \ldots, n-1$. Now, one can evaluate the probability amplitudes corresponding to the excitation or photon transition via the results (4-17). For instance, in the case of even $n=2 m$ with odd $m=2 l+1$, we have $x_{j}=\cos \frac{\pi j}{m} \neq 0$ for $j=0,1, \ldots, 2 m-1$, so that $x_{j}=x_{2 m-j}=-x_{m-j}$. Then, for the photon transition process, the results (5-21) and (5-22) lead to

$$
\begin{aligned}
& C_{i}(t) \simeq \frac{e^{-i \omega t} \cos t}{2 m} \sum_{j} \omega^{-i j}=e^{-i \omega t} \cos t \quad \delta_{i, 0} \\
& A_{i}(t) \simeq \frac{-i e^{-i \omega t} \sin t}{2 m} \sum_{j} \omega^{-i j}=e^{-i \omega t} \sin t \quad \delta_{i, 0}
\end{aligned}
$$

and

$$
\begin{gathered}
C_{i}(t) \simeq \frac{e^{-i \omega t}}{2 m} \sum_{j=0}^{2 m-1} e^{-2 i \xi t x_{j}} \omega^{-i j}=\frac{e^{-i \omega t}}{2 m}\left\{e^{-4 i \xi t}+(-1)^{i} e^{4 i \xi t}+2 \sum_{j=1}^{m-1} e^{-2 i \xi x_{j} t} \cos \frac{\pi j i}{m}\right\}, \\
A_{i}(t) \simeq \frac{-i e^{-i \omega t}}{2 m} \sum_{j} \frac{e^{-i \xi t x_{j}} \sin \left(\xi t x_{j}\right)}{\xi x_{j}} \omega^{-i j} \simeq 0
\end{gathered}
$$

for the asymptotic limits $\xi \ll$ and $\xi \gg$, respectively. The above relations indicate that for small hopping $\xi \rightarrow 0$, we have

$$
C_{0}(t) \simeq e^{-i \omega t} \cos t, \quad A_{0}(t) \simeq e^{-i \omega t} \sin t, \quad C_{i}(t)=A_{i}(t)=0 ; \text { for } i \neq 0,
$$

so that after time $t$, the initial state $|\psi(0)\rangle=|g, 2\rangle|g, 0\rangle \ldots|g, 0\rangle$ evolves as

$$
|\psi(t)\rangle \simeq e^{-i \omega t}(\cos t|g, 2\rangle+\sin t|e, 0\rangle)|g, 0\rangle \ldots|g, 0\rangle
$$

Therefore, the photon transition can not be achieved and a quantum correlation is generated between the atomic and photonic states of the first cavity. On the other hand, for large hopping $\xi \rightarrow \infty$, one obtains $A_{i}(t) \simeq 0$ for all times and

$$
C_{i=\text { odd }}(t) \simeq \frac{-i e^{-i \omega t}}{m}\left\{\sin 4 \xi t+2 \sum_{j=1}^{\frac{m-1}{2}} \sin \left(2 \xi x_{j} t\right) \cos \frac{\pi j i}{m}\right\}
$$


coupled cavities

$$
C_{i=\text { even }}(t) \simeq \frac{e^{-i \omega t}}{m}\left\{\cos 4 \xi t+2 \sum_{j=1}^{\frac{m-1}{2}} \cos \left(2 \xi x_{j} t\right) \cos \frac{\pi j i}{m}\right\}
$$

For example, for $n=6$ with $m=3$, we have $x_{0}=2, x_{1}=x_{5}=1, x_{2}=x_{4}=-1, x_{3}=-2$ and the above amplitudes are given by

$$
\begin{gathered}
C_{0}(t)=\frac{e^{-i \omega t}}{3}\{\cos 4 \xi t+2 \cos 2 \xi t\}, \\
C_{1}(t)=C_{5}(t)=\frac{-i e^{-i \omega t}}{3}\{\sin 4 \xi t+\sin 2 \xi t\}, \\
C_{2}(t)=C_{4}(t)=\frac{-i e^{-i \omega t}}{3}\{\cos 4 \xi t-\cos 2 \xi t\}, \\
C_{3}(t)=\frac{-i e^{-i \omega t}}{3}\{\sin 4 \xi t-2 \sin 2 \xi t\},
\end{gathered}
$$

Then, after time $T=\frac{3 \pi}{8 \xi}$, we obtain the probabilities associated with two photon transition from the first cavity to the other cavities as follows

$$
P_{0}(T)=\frac{2}{9} \simeq 0.22, \quad P_{1}(T)=P_{5}(T)=0.01, \quad P_{2}(T)=P_{4}(T)=0.05, \quad P_{3}(T)=\frac{5.76}{9} \simeq 0.64
$$

\section{The Hypercube network $Q_{d}$}

The Cayley graph of the direct product of groups $Z_{2}, d$ times (with the cartesian product of generating sets as a generating set) is the cartesian product of the corresponding Cayley graphs, which gives the hypercube graph $Q_{d}$ (called also the Hamming network $H(d, 2)$ ). Thus, the Cayley graph of the abelian group $G=\underbrace{Z_{2} \times Z_{2} \times \ldots \times Z_{2}}_{d \text {-times }}$ with the set of generators consisting of $n$ elements $(1,0,0, \ldots, 0),(0,1,0, \ldots, 0), \ldots,(0,0, \ldots, 0,1)$, is the hypercube network $Q_{d}$ with $n=2^{d}$ nodes and the adjacency matrix

$$
A=\sum_{i=1}^{d} I \otimes \ldots \otimes I \otimes \underbrace{\sigma_{x}}_{i-t h} \otimes I \ldots \otimes I .
$$

The irreducible characters of the group $G$ are direct products of the irreducible characters of the group $Z_{2}$, i.e., by denoting $k, l \in G$ in the binary representation $k \equiv k_{d-1} \ldots k_{1} k_{0}$ and $l \equiv l_{d-1} \ldots l_{1} l_{0}$, with $k_{i}, l_{i} \in\{0,1\}$ (so that $k=k_{0}+2^{1} k_{1}+\ldots+2^{d-1} k_{d-1}$ ), we have $\chi_{k}(l)=(-1)^{k \cdot l}$, where $k \cdot l=\sum_{i=0}^{d-1} k_{i} l_{i}$. Also, one can easily show that the eigenvalues of the 
adjacency matrix $A$ with the corresponding degeneracy degrees are given by $x_{i}=d-2 i$ and $D\left(x_{i}\right)=\frac{d !}{i !(d-i) !}$ for $i=0,1, \ldots, d$, respectively.

For instance, for $d=2$, we have $x_{0}=2, x_{1}=x_{2}=0$ and $x_{3}=-2$. Then, the Eq. (4-17) leads to

$$
\begin{gathered}
C_{i}(t)=\frac{e^{-i \omega t}}{4 \sqrt{1+4 \xi^{2}}}\left\{\sqrt{1+4 \xi^{2}}\left(e^{-2 i \xi t}+\chi_{3}(i) e^{2 i \xi t}\right) \cos t \sqrt{1+4 \xi^{2}}-2 i \xi\left(e^{-2 i \xi t}-\chi_{3}(i) e^{2 i \xi t}\right) \sin t \sqrt{1+4 \xi^{2}}+\right. \\
\left.\sqrt{1+4 \xi^{2}}\left(\chi_{1}(i)+\chi_{2}(i)\right) \cos t\right\} \\
A_{i}(t)=\frac{-i e^{-i \omega t}}{4 \sqrt{1+4 \xi^{2}}}\left\{\left(e^{-2 i \xi t}+\chi_{3}(i) e^{2 i \xi t}\right) \sin t \sqrt{1+4 \xi^{2}}+\sqrt{1+4 \xi^{2}}\left(\chi_{1}(i)+\chi_{2}(i)\right) \sin t\right\} .
\end{gathered}
$$

The above results are respectively simplified for the small and large hopping parameter $\xi$, as follows

$$
\begin{gathered}
C_{i}(t) \simeq \frac{e^{-i \omega t} \cos t}{4}\left\{e^{-2 i \xi t}+\chi_{3}(i) e^{2 i \xi t}+\chi_{1}(i)+\chi_{2}(i)\right\}, \\
A_{i}(t) \simeq \frac{-i e^{-i \omega t} \sin t}{4}\left\{e^{-2 i \xi t}+\chi_{3}(i) e^{2 i \xi t}+\chi_{1}(i)+\chi_{2}(i)\right\} ; \quad \text { for } \quad \xi \ll
\end{gathered}
$$

and

$$
\begin{gathered}
C_{i}(t) \simeq \frac{e^{-i \omega t}}{4}\left\{e^{-4 i \xi t}+\chi_{3}(i) e^{4 i \xi t}+\left(\chi_{1}(i)+\chi_{2}(i)\right) \cos t\right\} \\
A_{i}(t) \simeq \frac{-i e^{-i \omega t}}{4}\left(\chi_{1}(i)+\chi_{2}(i)\right) \sin t, \quad \text { for } \quad \xi \gg .
\end{gathered}
$$

By substituting $\chi_{1}(0)=\chi_{1}(2)=1, \quad \chi_{1}(1)=\chi_{1}(3)=-1, \chi_{2}(0)=\chi_{2}(1)=1, \quad \chi_{2}(2)=\chi_{2}(3)=$ -1 and $\chi_{3}(0)=\chi_{3}(3)=1, \quad \chi_{3}(1)=\chi_{3}(2)=-1$ in the above relations, the corresponding amplitudes are determined for every $i=0,1,2,3$.

\section{Conclusion}

In summery, quantum state transfer and entanglement generation between $n$ coupled atomcavity systems with uncolored Cayley interacting networks, was analyzed. By employing the excitation-photon conservation symmetry of the Hamiltonian and introducing some suitable 
'generalized' Fourier transformed basis states for the state space of the system, the corresponding Hilbert space was block-diagonalized with 2 dimensional blocks. Due to this useful reduction, the corresponding Shrödinger equation was solved exactly, and state transfer (excitation or photon transition between the atoms or the cavities) and entanglement generation between the atoms were discussed. The large and small hopping limits was discussed where, it was shown that for large hopping strength, the initially unentangled atoms remain effectively unentangled forever whereas for small hopping, the initially unentangled atoms become entangled and the initial photonic $W$-state oscillates between atomic and photonic $W$-states periodically.

\section{References}

[1] M. Christandl, N. Datta, A. Ekert, and A. J. Landahl, Phys. Rev. Lett. 92, 187902, (2004).

[2] M. Christandl, N. Datta, T. C. Dorlas, A. Ekert, A. Kay and A. J. Landahl, Phys. Rev. A 71, 032312, (2005).

[3] C. Facer, J. Twamley and J. Cresser, Phys. Rev. A 77, 012334, (2008).

[4] D. Burgarth and S. Bose, Phys. Rev. A 71, 052315, (2005).

[5] D. Burgarth and S. Bose, New J. Phys. 7, 135, (2005).

[6] M. H. Yung and S. Bose, Phys. Rev. A 71, 032310, (2005).

[7] M. H. Yung, Phys. Rev. A 74, 030303, (2006).

[8] M. A. Jafarizadeh and R. Sufiani, Phys. Rev. A 77, 022315, (2008).

[9] M. A. Jafarizadeh, et al., J. Phys. A: Math. Theor. 41, 475302, (2008).

[10] M A Jafarizadeh, et al., J. Stat. Mech. 05014, (2011). 
[11] Turchette, Q.A., Hood, C.J., Lange, W., Mabuchi, H., Kimble, H.J., Phys. Rev. Lett. 75, 4710 (1995); M. Brune et al., Phys. Rev. Lett. 77, 4887 (1996).

[12] Mattle, K., Weinfurter, H., Kwiat, P.G., Zeilinger, A., Phys. Rev. Lett. 76, 4656 (1996).

[13] M. Alexanian, Phys. Rev. A 83, 023814, 2011.

[14] A. Biswas and G. S. Agarwal, Phys. Rev. A 70, 022323 (2004).

[15] Cirac,J.I., Zoller,P., Kimble,H.J., Mabuchi,H., Phys. Rev. Lett. 78, 3221, (1997).

[16] M. Alexanian, Generation of entangled tripartite states in three identical cavities, arxiv: quant-ph/12034173.

[17] A.Ü.C. Hardal, Ö.E. Mstecaplioglu, J. Opt. Soc. Am. B 29, 1822-1828, (2012).

[18] R. Sufiani and A. Darkhosh, Generation of quantum entanglement between three level atoms via $n$ coupled cavities, arxiv: quant-ph/13033861.

[19] R. R. Puri, Mathematical methods of quantum optics, Springer, (2001).

[20] M. O. Scully and M. S. Zubairy, Quantum Optics, Cambridge University Press, (1997).

[21] Gordon James, Martin Liebeck,(1993), Representations and characters of groups ( Cambridge University Press, Cambridge).

[22] Andrei B. Klimov and Sergei M. Chumakov, (2009), A Group-Theoretical Approach to Quantum Optics (WILEY-VCH Verlag GmbH and Co. KGaA, Weinheim)

[23] A. Peres, Phys. Rev. Lett, 77, pp. 1413-1415, (1996).

[24] M. Horodecki, et. al, Phys. Lett. A, 223, pp. 1-8, (1996). 